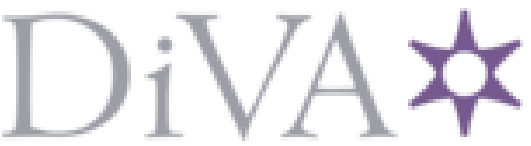

http://www.diva-portal.org

Postprint

This is the accepted version of a paper presented at 2019 IEEE Intelligent Vehicles Symposium (IV).

Citation for the original published paper:

Held, M., Flärdh, O., Roos, F., Mårtensson, J. (2019)

Implementation of an Optimal Look-Ahead Controller in a Heavy-Duty Distribution Vehicle

In: 2019 IEEE Intelligent Vehicles Symposium (IV) (pp. 2202-2207).

N.B. When citing this work, cite the original published paper.

Permanent link to this version:

http://urn.kb.se/resolve?urn=urn:nbn:se:kth:diva-257748 


\title{
Implementation of an Optimal Look-Ahead Controller in a Heavy-Duty Distribution Vehicle
}

\author{
Manne Held ${ }^{1,2}$, Oscar Flärdh ${ }^{1}$, Fredrik Roos ${ }^{1}$, and Jonas Mårtensson ${ }^{2}$
}

\begin{abstract}
Controlling the longitudinal movement of heavyduty vehicles based on optimal control can be a cost-efficient way of reducing their fuel consumption. Such controllers today mainly exist for vehicles in haulage applications, in which the velocity is allowed to deviate from a constant set-speed. For distribution vehicles, which is the focus of this paper, the desired and required velocity has large variations, which makes the situation more complex. This paper describes the implementation of an optimal controller in a real heavy-duty distribution vehicle. The optimal control problem is solved offline as a Mixed Integer Quadratic Program, which yields reference trajectories that are tracked online in the vehicle. Some important steps in the procedure of the implementation are, except for designing the controller: developing a positioning system for the test track where the experiments are performed, estimating the parameters of the resistive forces, and setting the velocity constraints. Simulations show a potential of $10 \%$ reduction in fuel consumption without increasing the trip time. Experiments are then performed in a Scania truck, with the optimal solution as reference for the existing cruise control functions in the vehicle. It is concluded that in order to verify the fuel savings experimentally, the low-level controllers in the vehicle must be modified such that the tracking error is decreased.
\end{abstract}

\section{INTRODUCTION}

Global warming is an urgent problem and the European Union has committed to reducing greenhouse gas emissions to $80-95 \%$ below 1990 levels by 2050 [1]. Since the transport sector accounts for as much as $22 \%$ of the $\mathrm{CO}_{2}$ emissions in the EU [2], the emissions in this sector must be reduced to comply with the commitment. An important part of the solution is to reduce the fuel consumption of vehicles. One way of doing so is by using more fuel-efficient control, which is the approach in this paper.

Fuel-efficient speed control of heavy-duty vehicles (HDV)s has been devoted a lot of attention in the last decade. The focus has been mainly towards haulage applications, i.e., highway driving, for which efficient commercial solutions exist today. Such controllers often allow the velocity to deviate from a fixed set-speed, which has resulted in for instance 3\% fuel reduction in [3]. By also choosing between coasting with a gear engaged and freewheeling, an additional $2 \%$ has been saved [4]. For environments where the velocity varies more than on highways, due to e.g. varying legal speed limits and curvature, such controllers do not exist to the same

Funding provided by Swedish Governmental Agency for Innovation Systems (VINNOVA) through the FFI program is gratefully acknowledged.

${ }^{1}$ Scania CV AB, 15187 Södertälje, Sweden.

${ }^{2}$ Department of Automatic Control and the Integrated Transport Research Lab, KTH Royal Institute of Technology, 10044 Stockholm, Sweden.

manneh@kth.se, oscar.flardh@scania.com,

fredrik.roos@scania.com, jonasl@kth.se extent. This is the main motivation for the work presented in this paper.

The problem of finding fuel-efficient speed and powertrain control has previously been solved in simulations in [5] and [6]. In these publications, the driving mission is formulated as an Optimal Control Problem (OCP) which is solved using different methods, such as Pontryagin's Maximum Principle offline and a Quadratic Program (QP) based Model Predictive Controller (MPC) online. This paper presents how such methods can be implemented in a real vehicle for performing experiments to measure fuel savings.

Speed controllers based on optimal control have been implemented and tested in heavy-duty vehicles for highway driving [7][8]. In [7], the optimal velocity profile is found offline and used as a reference for the speed controller. In [8], the optimal control is calculated online in the control unit of the vehicle. The potential of reducing the fuel consumption in HDVs forming platoons was investigated in [9]. One example of how real experiments are used in order to validate a simulation model which can be used for optimization is given in [10]. In [11], the optimal route and speed profile for a personal car in urban driving are calculated in the cloud and presented to the driver as a recommended speed.

Performing experiments with vehicles in applications with large variations in the velocity, in this paper referred to as distribution vehicles, is in some way more difficult than in highway driving. On highways, the vehicle can for most of the time follow the planned trajectory, since there are few obstacles, no traffic to yield for, and few vehicles driving as slow as the HDVs. For distribution vehicles on the other hand, there are pedestrians, intersections to yield at, and vehicles with low velocity. This makes experiments for fuel measurements less repetitive and more demanding in terms of safety. Therefore, the experiments described in this paper were chosen to be performed on a test track detached from public roads. The downside of this is that there are no publicly available map data for this test track, containing road grade and curvature. Because of this, an algorithm for positioning had to be developed.

This paper describes the development of an optimal control based longitudinal controller for performing experiment in a real heavy-duty distribution vehicle. A flow chart of the control design can be seen in Fig. 1. The main idea of the control framework is to formulate the driving mission as an OCP and solve it in order to generate a few look-up tables, such as a reference velocity, which are later tracked in an online controller. When formulating the OCP, legal speed limits and curvature are used together with actuator 


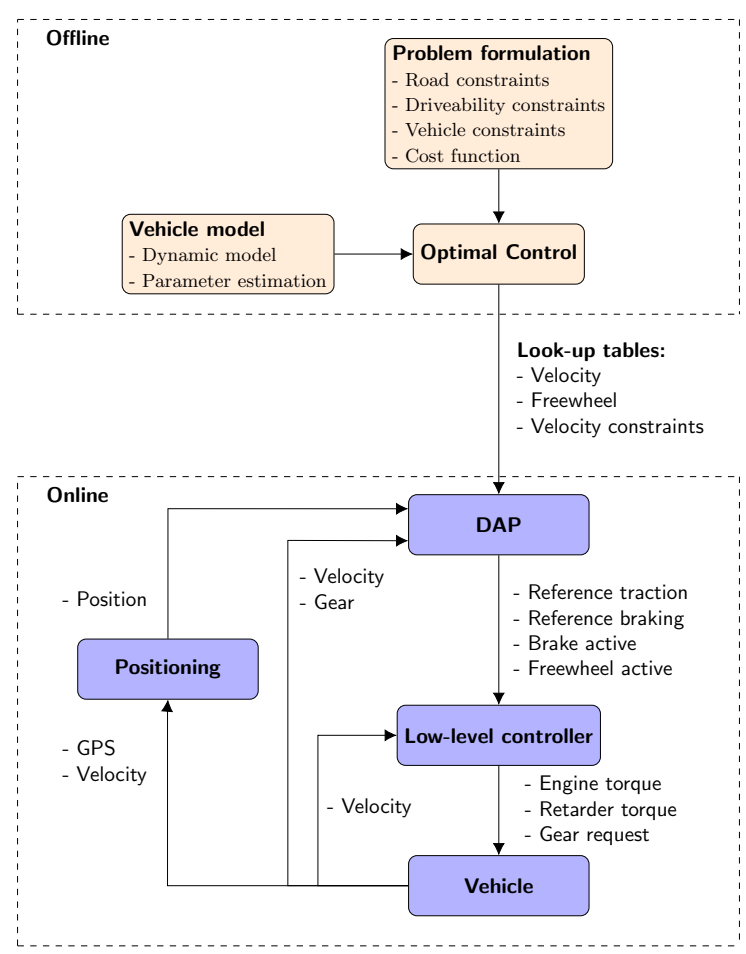

Fig. 1: Flow chart of the control design.

limitations in order to set an upper and a lower constraint for the velocity. The online controller, here referred to as Dynamic Active Prediction (DAP), uses an existing interface in the control unit of the vehicle in order to send the control signals to a low-level controller which controls the actuators of the vehicle. A GPS and the measured velocity are used in order to update the position of the vehicle which is used for finding the new control signals from the look-up tables. The block Low-level controller is an existing controller for generating engine torque, brake torque, and gear changes and is not modified in this work.

The main contributions of the paper are:

- An algorithm for automatically setting the velocity constraints for the OCP based on curvature and legal speed limits together with vehicle limitations and driveability considerations.

- A description of the procedure for developing the controller including parameter estimation, the positioning system, and the control framework.

\section{OFFLINE}

This section treats the parts in Fig. 1 denoted offline. First, the vehicle model is presented through the dynamic model and parameter estimation. Next, it is described how the velocity constraints are set and a cost function is introduced. Finally, it is described how these are combined such that the OCP can be formulated and solved.

\section{A. Vehicle model}

1) Dynamic model: The kinetic energy $K$ is used as the state variable as a function of position $s$. The dynamics of
TABLE I: Parameters for the vehicle and the environment.

\begin{tabular}{|l|c|}
\hline Parameter & Value \\
\hline$\rho$ - air density & $1.292 \mathrm{~kg} \cdot \mathrm{m}^{-3}$ \\
$a_{\text {max }, c}$ - maximum lateral acceleration & $1.5 \mathrm{~ms}^{-2}$ \\
$A_{f}$ - truck cross-sectional area & $\mathrm{m}^{2}$ \\
$c_{d}$ - air drag coefficient & {$[-]$} \\
\hline Estimated \& Measured Parameters & Value \\
\hline$A_{f} \cdot c_{d}$ - cross-sectional drag area & $6.8 \mathrm{~m}^{2}$ \\
$c_{r}$ - rolling resistance coefficient & 0.0067 \\
$r_{w}$ - wheel radius & $0.5 \mathrm{~m}$ \\
$m$ - vehicle mass & $30000 \mathrm{~kg}$ \\
\hline
\end{tabular}

the vehicle are given by

$$
\frac{d K(s)}{d s}=F_{t}(s)+F_{b}(s)+F_{a}(K(s))+F_{r}(s)+F_{g}(s)
$$

where $F_{t}$ is the tractive force, $F_{b}$ is the braking force, and $F_{a}(K(s))$ represents the air resistance such that

$$
F_{a}(K(s))=-\frac{\rho A_{f} c_{d} K(s)}{m}
$$

where $\rho$ is the air density, $A_{f}$ is the vehicle frontal area, $c_{d}$ is the air drag coefficient, and $m$ is the vehicle mass. The contribution from rolling resistance is given by

$$
F_{r}(s)=-m g c_{r} \cos (\alpha(s))
$$

where $c_{r}$ is the coefficient for the rolling resistance, $g$ is the gravitational constant and $\alpha$ is the road slope. The gravitational force $F_{g}(s)$ is given by

$$
F_{g}(s)=-m g \sin (\alpha(s)) .
$$

2) Parameter estimation: Before the experiment starts, a few parameters in the vehicle model (1) must be set. The vehicle mass $m$ is measured using a digital scale. Each axis is measured individually, and the masses are added. The product of the parameters for the frontal area and the drag coefficient $A_{f} c_{d}$ in (2) and the coefficient for the rolling resistance $c_{r}$ in (3) are estimated in a coast down test [12], [13]. The vehicle is given an initial velocity and freewheels for a section of known road grade. For such movement, both the tractive force $F_{t}$ and braking force $F_{b}$ in (1) are equal to zero, such that this equation can be written as

$$
a-\frac{F_{g}}{m}=g c_{r} \cos (\alpha(s))+\frac{1}{2} \frac{\rho A_{f} c_{d}}{m} v^{2} .
$$

Defining the vectors $y, X$, and $\beta$ where for element $i$

$$
\begin{aligned}
y_{i} & =a_{i}-\frac{F_{g, i}}{m}, \\
X_{i} & =\left[\begin{array}{ll}
g \cos \left(\alpha_{i}\right) & \frac{\rho v_{i}^{2}}{2 m}
\end{array}\right], \\
\beta & =\left[c_{r}, A_{f} c_{d}\right]^{T},
\end{aligned}
$$

the estimation is done using least squares such that the objective is

$$
\beta^{*}=\underset{\beta}{\operatorname{argmin}}\left((y-X \beta)^{T}(y-X \beta)\right)
$$




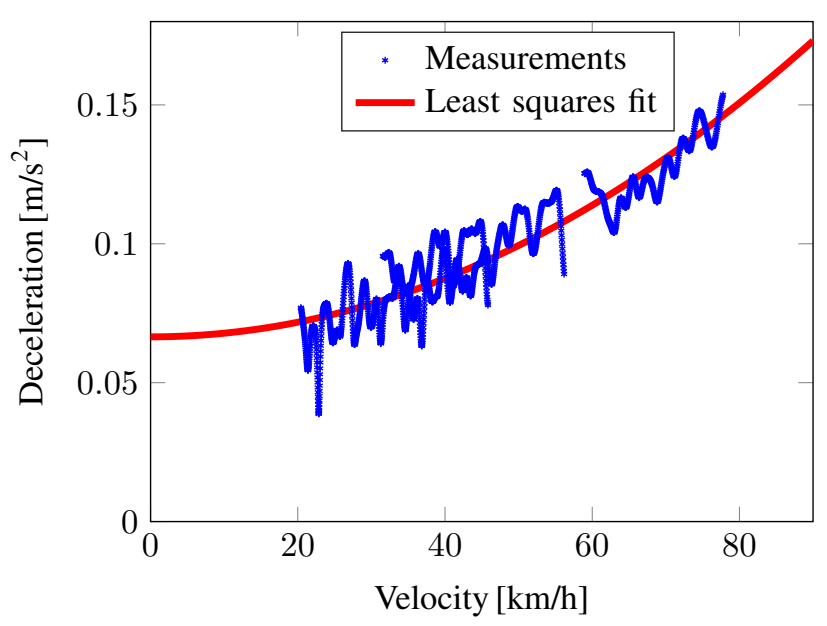

Fig. 2: Deceleration compensated for gravitational force during a coast down test together with the corresponding least squares fit. The test was performed with three different starting velocities.

which has the solution

$$
\beta^{*}=\left(X^{T} X\right)^{-1} X^{T} y
$$

Experimental results of deceleration compensated for gravity $y$, can be seen as a function of velocity together with the least squares fit in Fig. 2. The estimated parameters can be seen together with the parameters for the vehicle and the environment in Table I.

\section{B. Problem formulation}

The velocity of a vehicle is typically constrained by several factors. In this paper, the following factors are considered in order to set the constraints on the velocity:

1) Road constraints from legal speed limits and curvature.

2) Driveability constraints for adaption to a normal way of driving.

3) Maximum tractive and braking power and torque.

1) Road constraints: The test track is limited by standard signs for legal maximum speed, and the position and value of these are registered manually. The maximum velocity due to curvature $v_{\max , c}$ is set by using

$$
v_{\max , c}=\sqrt{r a_{\max , c}}
$$

where the curvature radius $r$ is measured as a function of position and the maximum lateral acceleration $a_{\max , c}$ is set based on driver comfort by manually driving on the track. These constraints can be seen together in Fig. 3.

2) Driveability constraints: The optimal control of a vehicle constrained by a maximum allowed velocity but without a minimum required velocity has been found to consist of long distances of coasting in order to avoid braking [5]. This behavior is fuel efficient, but can be unacceptable for the drivers and for trailing vehicles. A minimum allowed velocity is therefore used for driveability reasons. It is initially set as a percentage of the maximum value. However, if the maximum allowed velocity rapidly decreases, so will the minimum

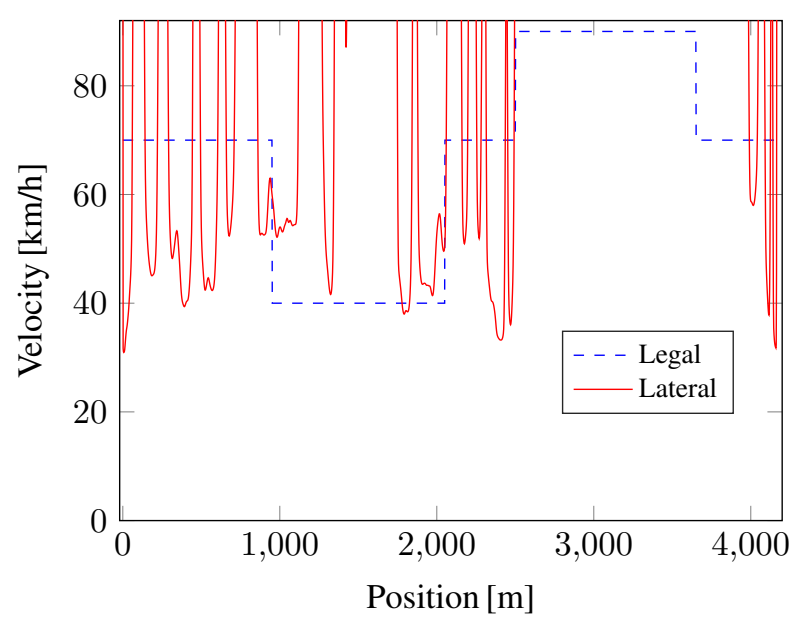

Fig. 3: Velocity constraints from legal speed limit and maximum lateral acceleration in curves.

allowed velocity. This will constrain the vehicle to extreme decelerations. To avoid this, the minimum allowed velocity is modified such that the vehicle is never forced to decelerate with more than some moderate value, which is motivated by the analysis in [5].

3) Vehicle constraints: The test track has sections of $8 \%$ uphills. Such sections can reduce the velocity of the HDV significantly. Setting the lower velocity constraint directly as in [5] might therefore result in infeasibility. To mitigate this, the lower constraint is modified in order to always contain a feasible solution. This is done by discretizing the constraint and for each step $k$ setting

$$
v_{\min }[k]=\min \left(v_{\min }[k], v_{\min }[k-1]+\Delta v_{\max }\right)
$$

where $\Delta v_{\max }$ is the acceleration given by maximum tractive power, taking road slope and resistive forces into account.

A similar problem can occur when the vehicle decelerates and/or drives in downhills. In this implementation, the retarder ${ }^{1}$ is used to reduce the velocity since this is the current design of the interface towards the low-level controller. Since the retarder has limitations in torque, it might be impossible not to violate the upper velocity constraint. Therefore, the upper constraint is modified such that it can always be fulfilled. This is done by setting

$$
v_{\max }[k]=\max \left(v_{\max }[k], v_{\max }[k+1]-\Delta v_{\min }\right)
$$

where $\Delta v_{\min }$ is the acceleration given by maximum braking, taking road slope and resistive forces into account. The final velocity constraints and the altitude can be seen in Fig 4.

\section{Optimal control problem}

The problem is formulated as a Mixed Integer Quadratic Program, with a boolean state variable for freewheeling. Kinetic energy is used as another state variable with dynamics given by (1) as a function of position discretized with $10 \mathrm{~m}$.

\footnotetext{
${ }^{1}$ A hydraulic system integrated into the output end of the gearbox which uses high-pressure oil as a braking medium.
} 

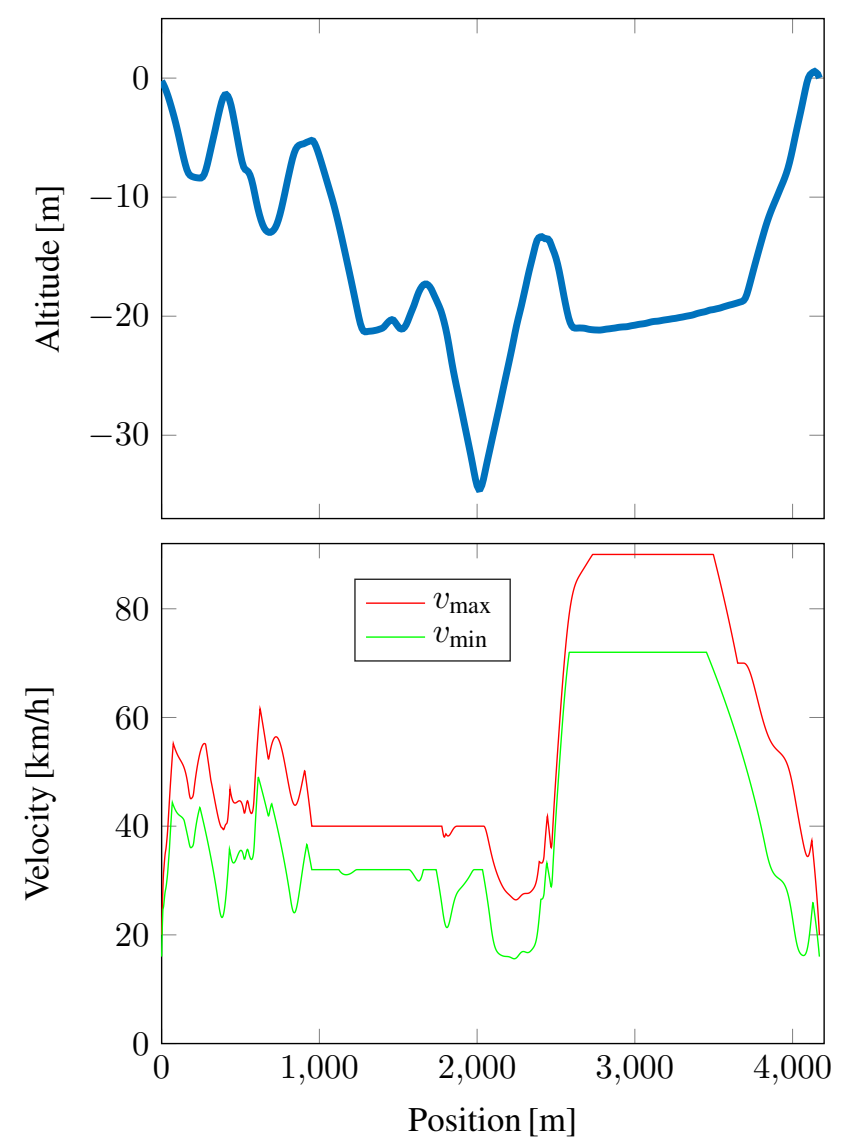

Fig. 4: Altitude of the test track and the upper and lower constraint on velocity used in the OCP.

The maximum and minimum allowed kinetic energy is at each position constrained by constant values. The constraints in maximum power are reformulated as constraints in force by multiplication with a first order Taylor approximation of the inverse of the velocity as a function of kinetic energy, see [5]. The cost function is a weighted sum of tractive energy, time consumption, and a penalty for gear changes.

\section{Online System}

This section first describes how measurements from the GPS and the velocity are combined to estimate the position along the track. Next, the online controller is described followed by comments on the low-level controller.

\section{A. Positioning}

The road grade ahead of the vehicle is normally obtained in Scania vehicles from maps from a third-party provider, together with a GPS. Since the experiments are performed on a test track, such maps do not exist. Instead, road grade measured by the vehicle is collected when it is manually driven around the track.

To be able to use the look-up tables derived offline, a positioning mechanism had to be developed. For this, the GPS signal is used together with the velocity of the vehicle. The GPS signal is given as longitude and latitude, which must be related to the position along the track. This is done by manually driving the driving cycle and collecting the GPS-signals as function of integrated velocity. The collected longitude and latitude can be transformed into $x$ and $y$ coordinates, and stored as functions of position in a table.

When using the online controller, the position from the GPS is calculated from the longitude and latitude signals transformed to $x$ and $y$ coordinates $x_{\mathrm{GPS}}$ and $y_{\mathrm{GPS}}$ by the scalar product

$$
\begin{aligned}
s_{G P S}( & \left.t-\Delta t_{\text {late }}\right)=\operatorname{pos}(\underline{x}, \underline{y}) \\
& +\frac{(\bar{x}-\underline{x}, \bar{y}-\underline{y})}{\|\bar{x}-\underline{x}, \bar{y}-\underline{y}\|} \cdot\left(x_{\mathrm{GPS}}-\underline{x}, y_{\mathrm{GPS}}-\underline{y}\right)
\end{aligned}
$$

where : is the coordinate value belonging to the previous table position, - the next table position and the function $\operatorname{pos}(x, y)$ gives the corresponding position. The GPS signals are sampled with a frequency of $\Delta t_{\mathrm{GPS}}$ with a latency of $\Delta t_{\text {late }}$. Both of these are assumed to be constant, and the time for measurement of the GPS signal can therefore be related to the arrival time of a previous signal. At the time of measurement, an integration of the velocity is started. At the time the GPS signal reaches the control unit, the true position measured by the GPS is then estimated by adding a dead-reckoning term such that

$$
s_{\mathrm{GPS}}(t)=s_{\mathrm{GPS}}\left(t-\Delta t_{\text {late }}\right)+\int_{t-\Delta t_{\text {late }}}^{t} v \mathrm{~d} t .
$$

The position calculated from the GPS signal is then used together with the integrated velocity since the last GPS signal to update the position of the vehicle. This is done such that

$$
\begin{aligned}
s(t)= & \alpha_{\mathrm{pos}}\left(s\left(t-\Delta t_{\mathrm{GPS}}\right)+\int_{t-\Delta t_{\mathrm{GPS}}}^{t} v \mathrm{~d} t\right) \\
& +\left(1-\alpha_{\mathrm{pos}}\right) s_{\mathrm{GPS}}(t)
\end{aligned}
$$

where $\alpha_{\text {pos }}=0.9$ is set empirically as a trade-off between drift and smoothness.

\section{B. Controller}

Solving the OCP yields look-up tables as functions of position, for velocity and for freewheeling. The controller is applied to an existing interface that enables the functionality in Scania Active Prediction [3]. The available input signals and types sent to this controller are:

- speed reference traction - continuous

- speed reference braking - continuous

- brake active - boolean

- gear request - integer.

The brake active signal is used in order to inform the lowlevel controller whether the velocity should be followed by traction or by braking. The braking speed reference is given by the upper velocity constraint $v_{\max }$. A hysteresis is used for the braking active signal such that the signal is deactivated when the velocity is $1 \mathrm{~km} / \mathrm{h}$ below the constraint. The continuous signals velocity reference and brake reference are obtained by linear interpolation of the positions in the lookup table. The gear request signal is used for freewheeling and is obtained by choosing the value belonging to the nearest of the positions in the look-up table. 

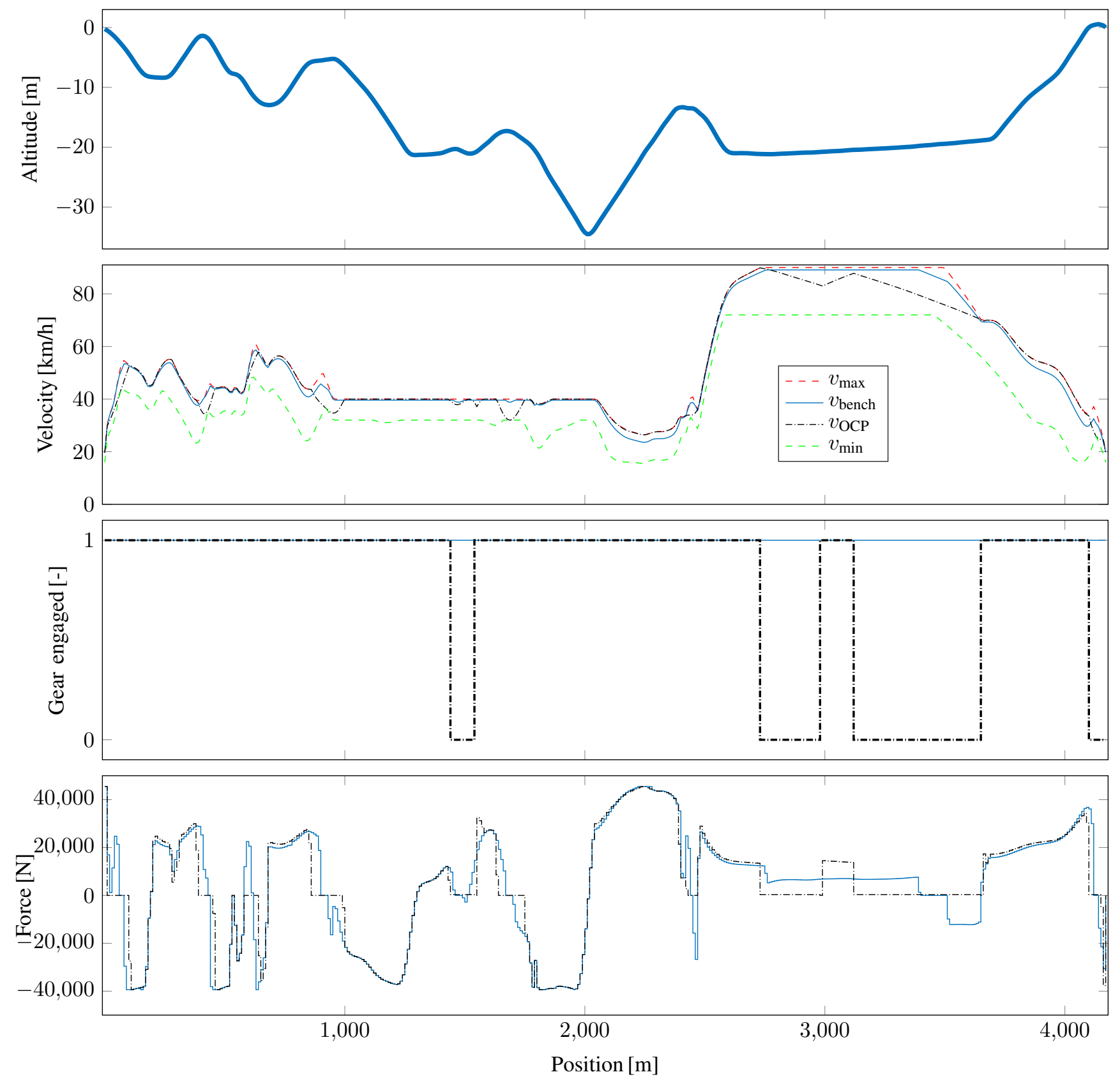

(a) Simulation results showing altitude, velocity, gear engaged, and control force. The velocity of the OCP is denoted $v_{\mathrm{OCP}}$ and the benchmark $v_{\text {bench. }}$

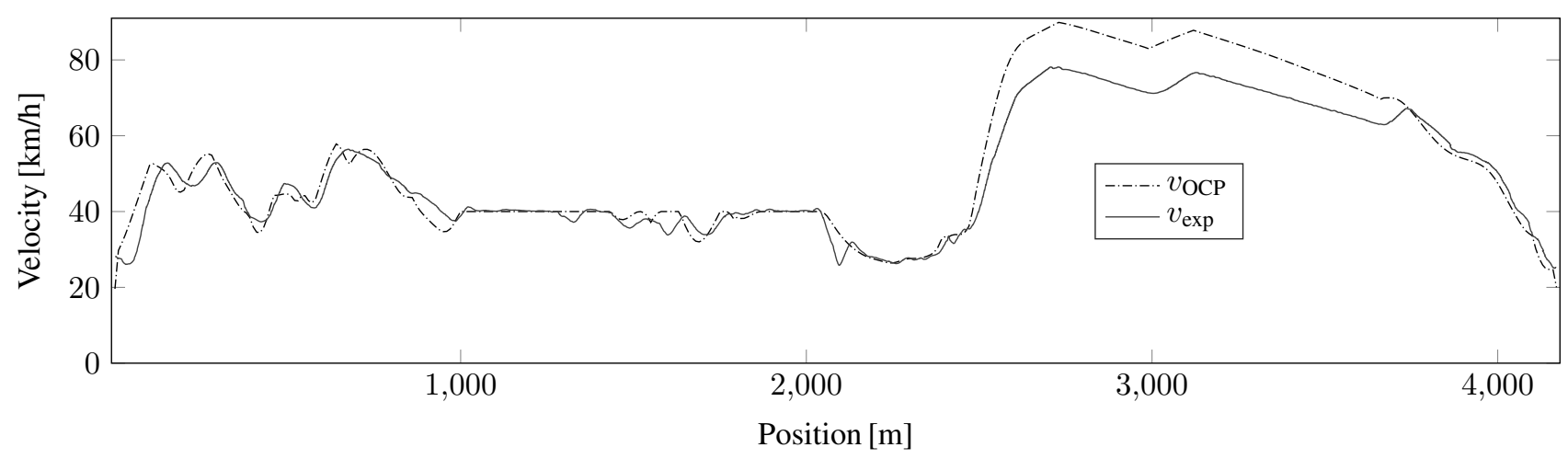

(b) Experimental results showing the reference to the speed controller $v_{\mathrm{OCP}}$ and the experimantal velocity of the HDV $v_{\text {exp }}$.

Fig. 5: Results from simulation and experiment. 


\section{Low-level controllers}

The low-level controllers used in the experiments are the existing controllers for velocity and for gear selection. The velocity controller is normally used for driving with small deviations from a constant speed reference and not for the varying trajectories derived in this paper. The default gear controller is used except for when freewheeling is requested. In such cases the default gear selection is overruled.

\section{RESUlTS}

The OCP was solved for generating reference trajectories for the online controller, but also for evaluating the potential fuel savings. To quantify these savings, the controller must be compared with a benchmark. For this purpose, the same optimal controller was chosen, but with much stricter constraints on the allowed deviations in velocity, deceleration and tractive power according to the discussion in Section II-B. These values can be seen together with the simulation results in Table II. The maximum allowed velocity for the benchmark was set lower than for the optimal controller in order to achieve a similar trip time.

The resulting trajectories of the optimal controller and the benchmark can be seen together in Fig. 5a. The optimal controller saves energy by coasting ahead of downhills, for instance at $400 \mathrm{~m}, 900 \mathrm{~m}$, and $1700 \mathrm{~m}$. Freewheeling is used three times, in all cases at positions with constant velocity constraints and small changes in altitude.

The trajectory from the OCP in the simulation was then used as a reference for the online controller. Resulting trajectory from the experiment in the real vehicle can be seen together with the simulated trajectory in Fig. 5b. As can be seen, the current low-level controller tracks the reference poorly. A major reason for this is that this controller is not designed for handling large variations in the reference, as is the case in this experiment. Instead, it is designed to settle smoothly to a fixed set-point.

An interesting result of the experiment would be to investigate the fuel consumption of the optimal controller compared with the benchmark controller, and whether or not the difference corresponds to the one found in the simulation. However, before such fuel measurements can be performed, the low-level controller must be adopted to this application in order to achieve smaller deviations between the reference and the experimental velocity.

\section{Summary AND Future Work}

In this paper, a driving mission representing distribution driving for an HDV has been formulated and solved as an OCP. The potential fuel savings have been calculated in simulation to be $10.3 \%$. The trajectory from the simulation has been used in experiments in a real HDV. In the next stage of this project, measurements of the fuel consumption of this vehicle will be performed. These tests will be performed with different settings on the constraints in the problem formulation, as done in the simulations. However, to obtain meaningful results of the fuel measurements, the low-level controller must be modified to track the reference better.
TABLE II: Constraints and results for the optimal controller and the benchmark. The maximum values are used for setting the upper velocity constraints and the minimum values for setting the lower velocity constraints.

\begin{tabular}{l|l|l} 
Settings & OCP & Benchmark \\
\hline Maximum velocity [\%] & 100 & 99 \\
Minimum velocity [\%] & 80 & 97 \\
Maximum power [\%] & 100 & 90 \\
Minimum power [\%] & 60 & 80 \\
Minimum deceleration [ms ${ }^{-2}$ ] & 0.40 & 0.55 \\
\hline Results & & \\
\hline Fuel consumption [\%] & 89.7 & 100 \\
Trip time [\%] & 99.8 & 100 \\
\hline
\end{tabular}

\section{REFERENCES}

[1] European Commission, "WHITE PAPER Roadmap to a Single European Transport Area Towards a competitive and resource efficient transport system, Tech. Rep. July, 2011. [Online]. Available: http://eurlex.europa.eu/ legal-content/EN/ALL/?uri=CELEX:52011DC0144

[2] — "EU Transport in Figures. Statistical Pocketbook," Tech. Rep., 2015. [Online]. Available: http://ec.europa.eu/transport/factsfundings/statistics/doc/2015/pocketbook2015.pdf

[3] Scania CV AB, "Scania Active Prediction, Tech. Rep. December, 2012. [Online]. Available: https:/www.scania.com/ group/en/event/pressroom-scania-active-prediction/

[4] _- "Scania utilises gravity to save fuel," Tech. Rep., 2013 [Online]. Available: https://www.scania.com/group/en/scania-utilisesgravity-to-save-fuel-3/

[5] M. Held, O. Flärdh, and J. Mårtensson, "Optimal Speed Control of a Heavy-Duty Vehicle in Urban Driving," IEEE Transactions on Intelligent Transportation Systems, no. 99, pp. 1-12, 2018.

[6] M. Henriksson, O. Flärdh, and J. Mårtensson, "Optimal Powertrain Control of a Heavy-Duty Vehicle Under Varying Speed Requirements," in 2017 IEEE 20th International Conference on Intelligent Transportation Systems (ITSC), Yokohama, 2017, pp. 1922-1927.

[7] E. Hellström, M. Ivarsson, J. Åslund, and L. Nielsen, "Look-ahead control for heavy trucks to minimize trip time and fuel consumption," Control Engineering Practice, vol. 17, no. 2, pp. 245-254, 2009.

[8] F. Mancino, "An embedded model predictive controller for optimal truck driving," Master Thesis, KTH Royal Institute of Technology, 2017.

[9] A. Alam, A. Gattami, and K. H. Johansson, "An experimental study on the fuel reduction potential of heavy duty vehicle platooning," in 2010 13th International IEEE Annual Conference on Intelligent Transportation Systems, Madeira Island, 2010, pp. 306-311.

[10] F. Lattemann, K. Neiss, S. Terwen, and T. Connolly, "The predictive cruise control: A system to reduce fuel consumption of heavy duty trucks," SAE Technical Paper 2004-01-2616, 2004.

[11] E. Ozatay, S. Onori, J. Wollaeger, U. Ozguner, G. Rizzoni, D. Filev, J. Michelini, and S. D. Cairano, "Cloud-Based Velocity Profile Optimization for Everyday Driving : A Dynamic-Programming-Based Solution," IEEE Transactions on Intelligent Transportation Systems, vol. 15, no. 6, pp. 2491-2505, 2014.

[12] I. Preda, D. Covaciu, and G. Ciolan, "Coast Down Test, Theoretical and Experimental Approach," in CONAT 2010 International Congress on Automotive and Transport Engineering, no. July, 2010, pp. 155 - 161. [Online]. Available: http://aspeckt.unitbv.ro/jspui/bitstream/ 123456789/7/1/CONAT20104030-Paper.pdf

[13] R. A. White and H. H. Korst, "The determination of vehicle drag contributions from coast-down tests," in 1972 Automotive Engineering Congress and Exposition. SAE International, feb 1972. [Online]. Available: https://doi.org/10.4271/720099 